\title{
Neue, von Herrn Dr. John Sahlberg auf seinen Reisen in Corfu, Palästina und Centralasien ge- sammelte Coleopteren.
}

Von Edm. Reitter in Paskau.

\section{Attumbra judaea n. sp.}

Nigra, nitida, parce pilosa, haud puberula, antennarum articulis duobus basalibus, prothoracis lateribus indeterminate pedibusque rufo-testaceis, elytris flavis, apice late nigris. Antennis thoracis basin superantibus, articulis 4-10 subquadratis, octavo parum minore, ultimo oblongo, fusco; ore palpisque testaceis. Capite, prothoraceque laevigatis, vix punctatis, hoc transverso, latitudine maxima pone medium sita, antrorsum magis attenuatis, angulis anticis deflexis, subrotundatis, posticis acuto-obtusis. Scutello glabro, nigro. Elytris subtiliter, vix seriatim punctatis, vix striatis, solum stria suturali leviter impressa, pilis erectis parce obsitis. Long.: $3 \mathrm{~mm}$.

Den bekannten Arten sehr ähnlich, von lucida und Josephinae durch den Mangel einer feinen anliegenden Grundbehaarung und zum grössten. Theile dunkle Fühler abweichend; von femoralis und subnuda durch gelbe Beine, von der ersteren durch Mangel einer feinen Grundbehaarung, von der letzteren durch glatten Thorax, dessen Seiten verwaschen rostroth gefärbt sind, und die spärlich punktirten Flügeldecken verschieden.

1 Exemplar von Bethlehem.

2. Catops cholevoides n. sp.

Oblongo-ovalis, dilute testaceus, nitidulus, pube fulva, subtili depressa dense vestitus, antennis elongatis tenuibus, dimidii corporis longitudine, articulis omnibus plus minusve subelongatis, prothorace transverso, densissime punctulato, coleopteris parum angustiore, lateribus leviter rotundato, pone medium (ante basin) magis dilatato, antrorsum magis rotundato, basi leviter rotundato, prope angulos posticos subrectos levissime sinuato, elytris oblongoovalibus, densissime punctulatis, obsolete striatis, stria suturalimagis impressa; pedibus gracilibus elongatis, femoribus tibiisque haud

Wiener Entomologische Ztitung, XIX. Jahrg., VIII. Heft (15. September 1900). 
incrassatis, tibiis anticis subrectis introrsum vix emarginatis intermediisque extus longe tenuiter spinosis, tarsis anticis, intermediis articulo primo longo cylindrico maris parum dilatato. Long.: $4 \mathrm{~mm}$.

Aus der Verwandtschaft des C. pallidus Mén., aber einer Choleva in Gestalt und Fühlerlänge gleich und als Catops nur durch das verdickte Basalglied der Mitteltarsen zu erkennen. Abweichend von den anderen Arten ist auch die lange Bedornung der Vorder- und Mittelschienen auf der Aussenseite.

1 Exemplar aus Transkaspien (Ahnger) von Dr. John Sahlberg mitgetheilt $\left(\sigma^{\top}\right)$.

Ich würde dieses Thier für Choleva lateritia Mén. halten, wenn es stumpfe Hinterwinkel des Halsschildes hätte.

3. Catops Sahlbergi 11. sp.

Dem Catops cholevoides täuschend ähnlich und durch nachfolgende Merkmale sicher specifisch unterschieden: Grösser, die Färbung ist gesättigter braunroth, Punktur und Behaarung sind noch viel dichter und feiner, die letztere gelblich, staubförmig; infolge der Dichte der Punktur und Behaarung ist die Oberseite fast matt, glanzlos; der Thorax ist etwas breiter, sehr wenig schmäler als die Basis der Flügeldecken, stärker quer, fast doppelt so breit als lang, die grösste Breite liegt ebenfalls etwas vor der Basis, Hinterwinkel und Basis sind gleich gebildet; hauptsächlich aber durch den Bau der Beine unterschieden; diese sind kräftiger gebaut und weniger lang, die Mittelschienen auch beim o deutlich gebogen, beim $\sigma^{\top}$ die Schenkel breiter, die Schienen, besonders die vorderen und mittleren, deutlich verdickt, die vorderen sind gerade, immer an der Basis mit einer deutlichen Ausrandung, die mittleren sind gebogen, die hintersten gerade, alle aussen lang, dünn bedornt. Die erweiterten Tarsen des $\sigma^{\top}$ wie bei der vorigen Art. L $0 \mathrm{ng}$. : $5 \mathrm{~mm}$.

Transkaspien: Ashabad. $\sigma^{\top}$ und o von Herrn Dr. John Sahlberg erbeutet.

\section{Agathidium Sahlbergi n. sp.}

Subglobosum, nitidum; ore, antennis (clava fere triarticulata nigra excepta) pedibusque testaceis, margine laterali sat lato, basali tenui, elytrorum apice indeterminate et sutura angustissima rufo-translucidis; antennis brevibus, articulo tertio solum leviter oblongo, articulis \%. et 8. parvulis, valde transversis; prothorace 
laevi, coleopterorum latitudine aut perparum angustiore, elytris fere laevibus, vix punctatis, stria suturali nulla, humeris subrecte truncatis. Long.: $1.8 \mathrm{~mm}$.

Vom Aussehen des A. haemorrhoum aber der Nahtstreifen fehlt. Gehört in die Gruppe des banaticum, nudum und aglyptoides; von allen diesen unterschieden durch die schwarze Fühlerkeule, die Färbung des Körpers und kurze, gedrungene Fühler. Clypeus nicht abgesetzt.

Aus Jaffa und Bethlehem, 2 Exemplare.

5. Cybocephalus aurocupreus n. sp.

Late et breviter subovatus, valde convexus, aurocupreus, nitidus, thoracis margine laterali elytrorumque limbo postico testaceis, antennis pedibusque fuscis. - Long.: $1 \mathrm{~mm}$.

Durch die kupferig goldene Färbung der ganzen Oberseite leicht kenntlich. Der Käfer ist kurz und überall hoch gewölbt, die Fühler und Beine sind dunkelpechfarben. Die Oberseite ist am Grunde sehr fein hautartig, gegen die Spitze viel deutlicher reticulirt und dazwischen kaum erkennbar punktirt.

2 Exemplare bei Michailowo (Transkaspien) von Dr. John Sahlberg aufgefunden.

\section{Dapsa opuntiae v. nov. Sahlbergi.}

Von der Stammform durch zwei schwarze, manchmal an der Naht zusammenlaufende Längsflecken unterschieden. Dadurch wird diese Art der denticollis und trimaculata recht ähnlich, aber sie entfernt sich von der ersteren durch sehr kurzen Zahn an den Vorderwinkeln, von der anderen durch diesen Zahn, von inornata Gorh. (caucasica M.) durch in der Mitte des Halsschildseitenrandes mangelnden zahnartigen Vorsprung.

Ebenfalls in einiger Anzahl von Dr. John S a h l be rg auf $\mathrm{C}$ orf u gesammelt.

\section{Trogoderma dichroa n. sp.}

Der Trog. villosula Duft. sehr ähnlich und nahe verwandt, aber etwas länger oval, die ganze Oberseite ist gelblich, weniger lang und weniger rauh, gleichmässig behaart, und durch die Färbung unterschieden. Schwarz, Flügeldecken mehr weniger dunkler; Unterseite schwarzbraun, die Beine rostbraun. Die Form des Halsschildes, dann die Punktur der Oberseite ist nahezu gleich mit der verglichenen Art. - Long.: $22.5 \mathrm{~mm}$.

Wiener Entomologische Ztitung, XIX. Jahrg., VIII. Heft (15. September 1900). 
Von T. amoenula Reitt. durch rauhere, gleichartige Behaarung, viel dichtere Punktur und dunkle Fühler unterschieden.

Turkestan: Issyk-kul. Von Dr. John S a h lberg zahlreich gesammelt.

\section{Platyscelis (Oodescelis) Sahlbergi n. sp.}

Eine neue kleine Art aus der ersten Gruppe der Oodescelis, wovon Dr. Seidlitz nur drei Arten kennt: melas, Heydeni und tibialis. Sie ist der tibialis sehr ähnlich, aber doppelt kleiner, und dreifach kleiner als melas; von den näherstehenden Heydeni und tibialis ausserdem durch die Bildung der männlichen Vorderschienen ausgezeichnet. Die innere Ausrandung derselben überragt nämlich hier weit die Mitte der Schienenlänge und der ganze Innenrand ist gelb behaart.

Oval, schwarz, Unterseite dicht und stark punktirt, Oberseite fett-glänzend. Fühler und Beine schlank, Kopf und Halsschild dicht und fein, sehr deutlich punktirt, Halsschild kaum ganz so breit als die Basis der Flügeldecken und um $1 / 3$ bis $1 / 4$ breiter als lang, von der Basis nach vorne verengt, die Seiten fein gerandet, dis Hinterwinkel fast spitzig, die Basis sehr flach ausgeschnitten, fast gerade. Flügeldecken feiner punktirt als der Thorax, beim o deutlieh bauchiger, ohne Streifen, oder Reihen. Prosternalfortsatz viel kürzer als bei den verwandten Arten, aber vortretend. Vorder- und Mittelfüsse stark erweitert, oben dunkel behaart. - L on g.: 7-8 $\mathrm{mm}$.

Turkestan: Verni, Almata. Von Dr. John Sahlberg gesammelt.

\section{Coccidula rufa v. nov. nigropunctata.}

Die rothgelben Flügeldecken haben hinter der Mitte je einen der Naht genäherten, scharf begrenzten, punktförmigen, schwarzen Flecken; machmal einen zweiten in der Mitte der Seitenrandnähe.

Ein Stück mit zusammen zwei Flecken besitze ich von Erivan (Russisch-Armenien), ein anderes mit vier Flecken wurde von Dr. Sahlberg bei Corfu gefunden. 


\section{$2 \mathrm{BHL}$ Biodiversity Heritage Library}

Reitter, Edmund. 1900. "Neue, von Herrn Dr. John SAHLBERG auf seinen Reisen in Corfu, Palästina und Centralasien gesammelten Coleopteren." Wiener entomologische Zeitung 19, 217-220.

https://doi.org/10.5962/bhl.part.3455.

View This Item Online: https://www.biodiversitylibrary.org/item/43789

DOI: https://doi.org/10.5962/bhl.part.3455

Permalink: https://www.biodiversitylibrary.org/partpdf/3455

\section{Holding Institution}

Smithsonian Libraries

\section{Sponsored by}

Smithsonian

\section{Copyright \& Reuse}

Copyright Status: NOT_IN_COPYRIGHT

This document was created from content at the Biodiversity Heritage Library, the world's largest open access digital library for biodiversity literature and archives. Visit BHL at https://www.biodiversitylibrary.org. 